OPEN ACCESS

Edited by:

Riccarda Granata,

University of Turin, Italy

Reviewed by:

Svetlana Lajic,

Karolinska Institutet (KI), Sweden

Jesús Devesa,

University of Santiago

de Compostela, Spain

*Correspondence:

Claudia Giavoll

claudia.giavoli@unimi.it

Specialty section:

This article was submitted to Neuroendocrine Science,

a section of the journal

Frontiers in Endocrinology

Received: 03 September 2020 Accepted: 02 December 2020

Published: 15 January 2021

Citation:

Giavoli C, lurlaro E, Morelli V, Rodari G, Ronchi A, Pietrasanta C, Pugni L,

Tubiolo D, Properzi P, Pesenti A,

Mantovani G, Ferrazzi E and Arosio $M$

(2021) Case Report:

Late-Onset Congenital Adrenal

Hyperplasia and Acute Covid-19

Infection in a Pregnant Woman:

Multidisciplinary Management.

Front. Endocrinol. 11:602535.

doi: 10.3389/fendo.2020.602535

\section{Case Report: Late-Onset Congenital Adrenal Hyperplasia and Acute Covid-19 Infection in a Pregnant Woman: Multidisciplinary Management}

\author{
Claudia Giavoli ${ }^{1,2 *}$, Enrico lurlaro ${ }^{3}$, Valentina Morelli ${ }^{1}$, Giulia Rodari ${ }^{1,2}$, Andrea Ronchi ${ }^{4}$, \\ Carlo Pietrasanta ${ }^{2,4}$, Lorenza Pugni ${ }^{4}$, Daniela Tubiolo ${ }^{5}$, Paolo Properzi ${ }^{5}$, \\ Antonio Pesenti ${ }^{5,6}$, Giovanna Mantovani ${ }^{1,2}$, Enrico Ferrazzi ${ }^{2,3}$ and Maura Arosio ${ }^{1,2}$ \\ ${ }^{1}$ Endocrinology Unit, Fondazione Istituto di Ricerca e Cura a Carattere Scientifico (IRCCS) Ca' Granda Ospedale Maggiore \\ Policlinico, Milan, Italy, ${ }^{2}$ Department of Clinical Sciences and Community Health, University of Milan, Milan, Italy, ${ }^{3}$ Unit of \\ Obstetrics and Gynecology, Department of Woman, Child and Neonate, Fondazione IRCCS Ca' Granda Ospedale Maggiore \\ Policlinico, Milan, Italy, ${ }^{4}$ Neonatology and Neonatal Intensive Care Unit (NICU), Fondazione IRCCS Ca' Granda Ospedale \\ Maggiore Policlinico, Milan, Italy, ${ }^{5}$ Department of Anesthesia, Critical Care and Emergency, Fondazione IRCCS Ca' Granda \\ Ospedale Maggiore Policlinico, Milan, Italy, ${ }^{6}$ Department of Pathophysiology and Transplantation, University of Milan, Milan, Italy
}

Background: The impact of the Covid-19 infection on patients with chronic endocrine disease is not fully known. We describe here the first case of a pregnant woman with Covid-19 acute infection and non-classical congenital adrenal hyperplasia (NCAH).

Case description: A woman at 36 weeks of gestation was referred to our Maternity Hospital for premature rupture of membranes (PROM). Her medical history was positive for $\mathrm{NCAH}$ on chronic steroid replacement till the age of 17 years (cortisone acetate and dexamethasone, both in the morning). At admission, her naso-oro-pharyngeal swab resulted positive for SARS-CoV-2. Due to hyperpyrexia and late preterm PROM, cesarean section was planned, and she was started on a $100 \mathrm{mg}$-bolus of hydrocortisone, followed by continuous infusion of $200 \mathrm{mg} / 24 \mathrm{~h}$. A female neonate in good clinical condition and with a negative nasopharyngeal Covid-19 swab was delivered. On second postpartum day, the mother was in good condition and was switched to oral steroid therapy. On third postpartum day she worsened, with radiological signs of acute pulmonary embolism. Orotracheal intubation and mechanical ventilation were started, and she was switched back to intravenous steroid therapy. On April 30, pulmonary embolism was resolved, and on May 13th she was discharged in good condition.

Conclusions: We report the first case of Covid-19 acute infection that occurred in latepregnancy in a woman with NCAH on chronic steroid replacement. The management of the patient in a reference center with early involvement of a multidisciplinary team granted prompt care and adequate protection for all the involved sanitary operators.

Keywords: pregnancy, Covid-19, congenital adrenal hyperplasia, adrenal, steroid replacement 


\section{BACKGROUND}

In Italy, Lombardy was one of the first and worst hit regions by the novel coronavirus disease (Covid-19). Here we describe the first case of acute Covid-19 infection in a pregnant woman affected with late-onset congenital adrenal hyperplasia.

\section{CASE DESCRIPTION}

On April 6, 2020, a 39 years-old pregnant woman at 36 weeks of gestation was referred to our Maternity Hospital for premature rupture of membranes (PROM). In the previous days, she had suffered from hyperpyrexia up to $38.6^{\circ} \mathrm{C}$, easily managed by paracetamol. Prior to admission, the naso-oro-pharyngeal swab performed in the emergency room resulted positive for SARS-CoV2 at rRT-PCR assay. She weighed $97 \mathrm{~kg}$, her height was $166 \mathrm{~cm}$, blood pressure was $128 / 74 \mathrm{mmHg}$, O2sat was $100 \%$, and respiratory rate was $16 \mathrm{bpm}$. Medical history was positive for late onset congenital adrenal hyperplasia due to 21-hydroxilase deficiency (NCAH) diagnosed at the age of 17 because of elevated basal 17hydroxyprogesterone (17OHP) concentrations at the investigations performed for hyperandrogenism and alopecia. Since then, she was on steroid replacement therapy (cortisone acetate $12.5 \mathrm{mg}$ plus dexamethasone $0.75 \mathrm{mg}$ per day, both in the morning). She had two previous pregnancies, one six years earlier, delivered at term and one first trimester miscarriage. Glucocorticoid dosage had never been adjusted. Cesarean section was planned due to persisting hyperpyrexia and late preterm PROM. She was started on a 100 mg-bolus of hydrocortisone, followed by continuous infusion of 200 $\mathrm{mg} / 24 \mathrm{~h}$ as indicated to avoid adrenal crisis in surgical intervention of chronic adrenal insufficient patients (1-3). She was delivered by cesarean section under spinal anesthesia, provided its positive influence on cardiopulmonary function (4).

At 36 weeks of gestational age, a female neonate was delivered. She was in good clinical condition, and her nasopharyngeal swab for SARS-CoV-2 by rRT-PCR was negative.

On second postpartum day, the mother was in good condition and was switched from intravenous to oral supra-physiological steroid therapy (cortisone acetate $25+12.5+12.5 \mathrm{mg} /$ day); at that time since her temperature was $37.5^{\circ} \mathrm{C}$, she was started on full dose low molecular weight heparin. On the third day her clinical conditions progressively worsened, with fever $>39^{\circ} \mathrm{C}$ associated with respiratory symptoms requiring $\mathrm{O} 2$ support. Thus, therapy with hydroxychloroquine and azithromycin was started. Accordingly, cortisone acetate was increased to overall 75 mg/day. A Chest Computed Tomography (CT) showed signs of acute pulmonary embolism, along with extensive ground-glass opacifications involving both the lung parenchyma (Figure 1) and laboratory tests showed high D-Dimer $(4,136 \mu \mathrm{g} / \mathrm{L}, \mathrm{nv}<500$

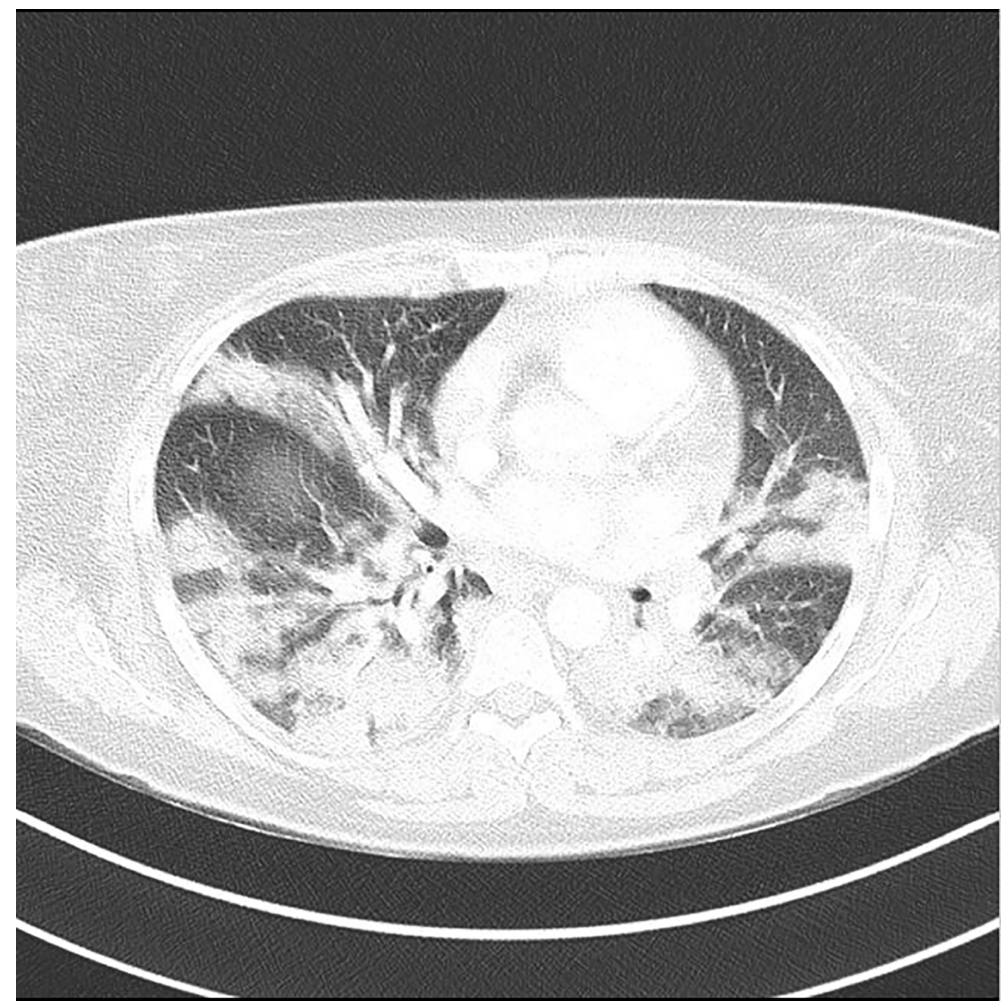

FIGURE 1 | Chest CT showing signs of acute pulmonary embolism, along with extensive ground-glass opacifications involving both the lung parenchyma. 
$\mu \mathrm{g} / \mathrm{L})$ and CRP $(11.22 \mathrm{mg} / \mathrm{dl}, \mathrm{nv}<0.05 \mathrm{mg} / \mathrm{dl})$. Her conditions rapidly worsened so she was moved to the Intensive Care Unit (ICU); orotracheal intubation along with mechanical ventilation were started, and she was promptly switched back to intravenous steroid therapy (Hydrocortisone, $50 \mathrm{mg}$ every $6 \mathrm{~h}$ iv).

This aggressive respiratory and medical support allowed her to recover and, after the first week, to improve significantly her general condition. On April 30, pulmonary embolism was completely resolved at CT scans. Finally, on May 13th, she was discharged in good condition.

At the beginning of May, hydrocortisone was progressively reduced and switched to oral route (cortisone acetate $12.5+$ $12.5+6.25 \mathrm{mg}$ ) to continue monitoring at our tertiary outpatient's clinics.

\section{DISCUSSION}

We report the first case of Covid-19 acute infection that occurred in late-pregnancy in a woman with NCAH on chronic steroid replacement.

Non-classic congenital adrenal hyperplasia is an autosomal recessive disorder due to a deficiency of 21-hydroxylase, an essential enzyme for the adrenal synthesis of cortisol and aldosterone. NCAH patients typically have $20-70 \%$ residual enzyme activity, enough to maintain a normal electrolyte homeostasis by aldosterone, but with a variable deficiency of cortisol, reduced pituitary feed-back, and increased ACTH and adrenal androgen production. By suppressing $\mathrm{ACTH}$ production, glucocorticoids can normalise the excessive androgen production and can be used in symptomatic patients to increase fertility and during pregnancy (3, 5-7). However, during pregnancy and especially in $\mathrm{NCAH}$, most experts do not recommend dexamethasone, a category $\mathrm{C}$ drug that passes the placenta and has potential adverse effects on both mother and fetus $(3,6,8)$.

In the absence of data about her native adrenal function, we managed successfully the patient as adrenal insufficient according to the current Guidelines (1-3). Definitely, this could be assumed at least as resulting from long-term (more than 20 years) high dose steroid treatment.

The COVID-19 infection, in this case probably of familiar origin, affected many young women between March and April in Lombardy, where about $9 \%$ of cases occurred in women between 30 and 39 years (9). However, the disease was particularly severe in our patient requiring a long recovery in ICU with a particularly challenging management, since the infection spread in late pregnancy.

Indeed, a systematic review including 538 pregnant women with Covid-19 infection reported approximately the same rate of ICU admission as in the non-pregnant population, but an increased risk of preterm and cesarean delivery (10).

To frame the situation, in the period between March $1^{\text {st }}$ and April $30^{\text {th }}, 852$ women delivered in Mangiagalli Obstetrics Unit, a high-risk maternity center in Milan, and one of the six Covid19 hubs identified in Lombardy to centralize care of pregnant patients affected by SARS Co-v 2 infection (11). Among these, 25 had confirmed COVID-19 infection (3\%). In the Covid-19 group, 11 (44\%) women delivered by cesarean and 14 (56\%) vaginally. Only two women were admitted to the ICU and one in the sub-intensive Unit. The patient here described was the only one requiring mechanical ventilation.

It is possible to hypothesize that the long-term steroid therapy may have contributed to cause either a major risk of infection or a greater clinical picture severity. Concordant to this hypothesis, a recent paper reported an increased risk of lower respiratory tract infections in $\mathrm{CAH}$ patients on chronic glucocorticoids therapy, not only compared to general population, but also to $\mathrm{CAH}$ patients not treated with glucocorticoids, thus enlightening that non-physiological delivery of glucocorticoid replacement may represents a risk factor for infections development (12). Moreover, as in other critical illnesses, Covid-19 pneumonia can affect residual adrenal function through cytokine release, not only worsening the outcome but also raising the risk either of medical complications as well as of progressing to worse critical stages (13). Few data on Covid-19 infection spread and course in patients with adrenal disorders are available so far. A recent survey published by our group reported a similar rate and severity of Covid-related symptoms in adrenal insufficient patients and in controls (14). In this study CAH patients were excluded because of the possible detrimental role both of androgen excess and of not fully physiological steroid therapy.

While present report was under review, a first case of SARSCoV-2 infection in a 5-week-old infant with adrenal insufficiency secondary to $\mathrm{CAH}$ was published (15). The authors underline the positive clinical course of SARS-CoV-2, even in the presence of underlying adrenal insufficiency and suggest that even though hydrocortisone was started to treat adrenal insufficiency, it may have also contributed to improve response to SARS-CoV2 infection.

Similarly, in the woman here reported, hydrocortisone 200 $\mathrm{mg} /$ die (1) has been sufficient not only to prevent acute adrenal crisis but also to reach disease remission (16).

From the pandemic outspread up to now, plenty of literature has tried to widen the knowledge of this novel Coronavirus, and the medical approach is continuously evolving.

For instance, considering the latest data about the use of dexamethasone $(17,18)$, it is possible to hypothesize that an earlier use of higher corticosteroid dose might have prevented the worsening of her clinical conditions.

Similarly, even though no consensus is still available, more recent case series or reports about postpartum patients with Covid-19 infection tend to support the benefits of thromboprophylaxis (19). Moreover, in the last few months the role of immune response on the pathogenesis of Covid-19 complications pointed out the possible benefit of immunemodulators as adjuvant therapy (20). In this context, reports about the use of melatonin suggest its possible beneficial effects in anti-inflammation, anti-oxidation, immune response regulation, as demonstrated in respiratory disorder models induced by infections. Thus, even though the direct evidence of melatonin application in Covid-19 is not fully clear, given also 
its high safety profile, these suggestions may prompt its use in Covid-19 patients (21).

Treating an acute Covid-19 infection, spread in late pregnancy and at the beginning of Covid-19 pandemic, was particularly challenging not only for the adrenal condition, but also for all the uncertainties on best therapeutic approaches.

Undoubtedly, the management of the patient and the neonate in a reference center with early involvement of a multidisciplinary team, through application of the best medical knowledges available at that time, granted prompt care for them and adequate protection for all the involved sanitary operators.

\section{DATA AVAILABILITY STATEMENT}

The raw data supporting the conclusions of this article will be made available by the authors, without undue reservation.

\section{REFERENCES}

1. Woodcock T, Barker P, Daniel S, Fletcher S, Wass JAH, Tomlinson JW, et al. Guidelines for the management of glucocorticoids during the peri-operative period for patients with adrenal insufficiency. Anaesthesia (2020) 75:654-63. doi: 10.1111/anae.14963

2. Bornstein SR, Allolio B, Arlt W, Barthel A, Don-Wauchope A, Hammer GD, et al. Diagnosis and Treatment of Primary Adrenal Insufficiency: An Endocrine Society Clinical Practice Guideline. J Clin Endocrinol Metab (2016) 101:364-89. doi: 10.1210/jc.2015-1710

3. Speiser PW, Arlt W, Auchus RJ, Baskin LS, Conway GS, Merke DP, et al. Congenital Adrenal Hyperplasia due to Steroid 21-Hydroxylase Deficiency: An Endocrine Society Clinical Practice Guideline. J Clin Endocrinol Metab (2018) 103:4043-88. doi: 10.1210/jc.2018-01865

4. Peng PWH, Ho P-L, Hota SS. Outbreak of a new coronavirus: what anaesthetists should know. Br J Anaesth (2020) 124:497-501. doi: 10.1016/j.bja.2020.02.008

5. Nordenstrom A, Falhammar H. MANAGEMENT OF ENDOCRINE DISEASE: Diagnosis and Management of the Patient with Non-Classic CAH Due to 21-hydroxylase Deficiency. Eur J Endocrinol Metab (2019) 180:R127-45. doi: 10.1530/EJE-18-0712

6. van't Westeinde A, Karlsson L, Nordenström A, Padilla N, Lajic S. FirstTrimester prenatal Dexamethasone Treatment is Associated with Alterations in Brain Structure at Adult Age. J Clin Endocrinol Metab (2020) 105:1-12. doi: 10.1210/clinem/dgaa340

7. Parsa AA, New MI. Steroid 21-hydroxylase Deficiency in Congenital Adrenal Hyperplasia. J Steroid Biochem Mol Biol (2017) 165:2-11. doi: 10.1016/ j.jsbmb.2016.06.015

8. Bothou C, Anand G, Li D, Kienitz T, Seejore K, Simeoli C, et al. Current management and outcome of pregnancies in women with adrenal insufficiency: experience from a multi-center survey. Clin Endocrinol Metab (2020). doi: 10.1210/clinem/dgaa266

9. Istituto Superiore di Sanità. Coronavirus(2020). Available at: https://www. epicentro.iss.it/coronavirus/ (Accessed May 20, 2020).

10. Huntely BJ, Huntley ES, Di Mascio D, Chen T, Berghella V, Chauhan SP. Rates of Maternal and Perinatal Mortality and Vertical Transmission in Pregnancies Complicated by Severe Acute Respiratory Syndrome Coronavirus 2 (SARS-Co-V-2) Infection: A Systematic Review. Obstet Gynecol (2020). doi: 10.1097/AOG.0000000000004010

11. Ferrazzi EM, Frigerio L, Cetin I, Vergani P, Spinillo A, Prefumo F, et al. COVID-19 Obstetrics Task Force, Lombardy, Italy: Executive management summary and short report of outcome. Int J Gynaecol Obstet (2020) 149:3778. doi: 10.1002/ijgo.13162

12. Tresoldi AS, Sumilo D, Perrins M, Toulis KA, Prete A, Reddy N, et al. Increased Infection Risk in Addison's Disease and Congenital Adrenal

\section{ETHICS STATEMENT}

Ethical review and approval was not required for the study on human participants in accordance with the local legislation and institutional requirements. The patients/participants provided their written informed consent to participate in this study. Written informed consent was obtained from the individual(s) for the publication of any potentially identifiable images or data included in this article.

\section{AUTHOR CONTRIBUTIONS}

CG, EI, VM, CP, AR, CP, LP, DT, and PP performed treatment and follow-up of the patients and the newborn. CG, VM, and GR collected clinical data and prepared the manuscript. AP, GM, EF, and MA performed the critical revision of the manuscript. All authors contributed to the article and approved the submitted version.

Hyperplasia. J Clin Endocrinol Metab (2020) 105:418-29. doi: 10.1210/ clinem/dgz006

13. Teblick A, Peeters B, Langouche L, Van den Berghe G. Adrenal function and dysfunction in critically ill patients. Nat Rev Endocrinol (2019) 15:417-27. doi: 10.1038/s41574-019-0185-7

14. Carosi G, Morelli V, Del Sindaco G, Serban AL, Cremaschi A, Frigerio S, et al. Adrenal insufficiency at the time of COVID-19: a retrospective study in patients referring to a tertiary centre. J Clin Endocrinol Metab (2020). doi: 10.1210/clinem/dgaa793

15. Azouz H, Gerrits P, Surhigh J, Kalladi Puthanpurayil SK. COVID-19 in an Infant with Congenital Adrenal Hyperplasia: A Case Report. Global Pediatr Health (2020) 7:1-3. doi: 10.1177/2333794X20958933

16. Isidori AM, Arnaldi G, Boscaro M, Falorni A, Giordano C, Giordano R, et al. COVID-19 infection and glucocorticoids: update from the Italian Society of Endocrinology Expert Opinion on steroid replacement in adrenal insufficiency. J Endocrinol Invest (2020). doi: 10.1007/s40618-020-01266-w

17. Johnson RM, Vinetz JM. Dexamethasone in the management of Covid -19. BMJ (2020) 370:m2648. doi: 10.1136/bmj.m2648

18. Mukhtar H, Ahmed MH, Hassan A. Dexamethasone for the Treatment of Coronavirus Disease (COVID-19): a Review. SN Compr Clin Med (2020). doi: 10.1007/s42399-020-00610-8

19. Benhamou D, Keita H, Ducloy-Bouthors ASCARO working group. Coagulation changes and thromboembolic risk in COVID-19 obstetric patients. Anaesth Crit Care Pain Med (2020) 39:351-3. doi: 10.1016/j.accpm.2020.05.003

20. Moghadam SO. Review on currently available potential therapeutic options for COVID-19. Int J Gen Med (2020) 13:443-67. doi: 10.2147/IJGM.S263666

21. García Garcia I, Rodriguez-Rubio M, Rodríguez Mariblanca A, Martínez de Soto L, Díaz García L, Monserrat Villatoro J, et al. A randomized multicenter clinical trial to evaluate the efficacy of melatonin in the prophylaxis of SARSCoV-2 infection in high-risk contacts (MeCOVID Trial): A structured summary of a study protocol for a randomised controlled trial. Trials (2020) 21(1):46. doi: 10.1186/s13063-020-04436-6

Conflict of Interest: The authors declare that the research was conducted in the absence of any commercial or financial relationships that could be construed as a potential conflict of interest.

Copyright (c) 2021 Giavoli, Iurlaro, Morelli, Rodari, Ronchi, Pietrasanta, Pugni, Tubiolo, Properzi, Pesenti, Mantovani, Ferrazzi and Arosio. This is an open-access article distributed under the terms of the Creative Commons Attribution License (CC BY). The use, distribution or reproduction in other forums is permitted, provided the original author(s) and the copyright owner(s) are credited and that the original publication in this journal is cited, in accordance with accepted academic practice. No use, distribution or reproduction is permitted which does not comply with these terms. 